\title{
Six Reasons Why Mary Fischer Hated Breastfeeding (and Six Things I Wish We Could Have Said)
}

Share this:

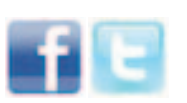

I was recently surfing my way through social media and I happened upon a blog post entitled "Six Reasons I Hated Breastfeeding and Would Never Do It Again." I didn't post her article on my Facebook page, but I wanted to hear more about her experience. She breastfed for two weeks and then stopped. And she didn't just hate breastfeeding-she loathed it. I wondered why. Why hadn't things worked out for her? How could we have supported her better?

In many ways, Mary's experience highlights the problems that new mothers encounter in the U.S. If we want to prevent premature weaning, we need to listen to women who decide to wean and find out what happened to them once they left the hospital. The following are Mary Fischer's reasons for why she hated breastfeeding. I think we would hear similar stories from other new mothers.

1. "It's all I did-OMG." Mary noted that her baby took an hour to feed and the baby was on her $24 / 7$. It was overwhelming.

2. “I needed sleep." Nighttime feedings also overwhelmed her. It helped when her husband could handle some of these.

3. "It hurt like hell." Breastfeeding hurt and was making her cry-and she was already crying a lot.

4. "My baby was hungry." She worried that her baby wasn't getting enough to eat and was fussy as a result. She reported that his demeanor completely changed once he was bottle-fed and had a full belly.

5. "It made me feel more isolated." Breastfeeding in public was out of the question for her. She felt like she couldn't go out and was very isolated and lonely.

6. "I wanted my body back." After being pregnant for so many months, she was happier once she had her body back.

Mary has outlined some common reasons mothers cite for why they stop breastfeeding. The following is what I wish we could have said to Mary before she got to that point.

1. Based on the history Mary reported in her blog post, most of us would recognize that something was not working with her latch. It hurt, but the baby was

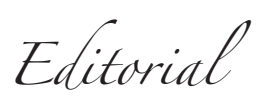

eating "all the time," and the mother reported that her baby seemed hungry. If her baby was gaining well, we could have reassured her and talked with her about how to tell if her baby was getting enough. If I were to see her, my immediate goal would be to address her nipple pain. Something clearly wasn't working and I would want to make sure to address her pain tout sweet.

2. Mary Fischer was clearly overwhelmed with the demands of new motherhood. And should we be surprised by her reaction? No! Generally speaking, our culture does an extremely poor job of supporting new mothers. Mothers leave the hospital and step right back in their lives. Rather than having lots of good support from people who come and bring them food, tidy their houses, and sit and talk with them, American mothers are expected to do all the things they normally do plus be up making snacks for everyone who comes to see the baby. No wonder she felt "tied down" by having to hold her baby all the time. She was expected to be "doing something productive" with her time. What could be more productive than what she was already doing? Yet she seemed to feel that she needed to do "more." When she couldn't meet our society's unrealistic expectations, she felt like she was failing.

3. On a related point, we often do a poor job of preparing mothers for the realities of parenting a baby. Postpartum is hard. It is intense. But it doesn't last forever. Helping mothers to understand that holding the baby is a way to transition him from womb to world often makes it more tolerable. Suzanne Colson explains to mothers that the baby has just come from an environment where he was "held" in utero 24/7. It's not unreasonable for a baby to want to be held a lot as they transition to life outside the womb.

4. It's not hard to understand why she felt so isolated. Unfortunately, we still live in a culture where it is not always safe to nurse a baby in public. The thought of being out and about and still needing to nurse can be a significant barrier for many new mothers. It only takes hearing about one mother forced off a plane or asked to leave a restaurant for many other new mothers to decide that they will not risk it. 
As a result, many women start bottle-feeding because they are finally "broken" by the social isolation. Remember, social isolation is often used as a form of torture. It is not surprising that mothers cannot endure it. And they really shouldn't have to.

5. Mary Fischer had also reached her breaking point regarding sleep. The fact that breastfeeding hurt would be enough to keep her from sleeping. If we had been able to address that issue, she would have gotten more rest while breastfeeding. Sleep deprivation is also another form of torture, and painful breastfeeding was definitely adding to her sleeplessness. It doesn't have to be this way eithernor should it have been.

6. As a final point, we shouldn't overlook the role of postpartum depression in her experience. Mary's pain, sleep problems, and social isolation are practically a recipe for depression. She notes this herself, "But then when [breastfeeding] pretty much took over my life and made me even more exhausted, overwhelmed, and depressed than I already was, I realized that breastfeeding was never going to be my cup of tea."

If you've ever have a day where you've wondered if the work you do makes a difference, please remember Mary's story. If not for your efforts, there would be thousands of other mothers who would report a similar tale. Instead, you help mothers every day get the best possible start to parenting by modeling compassion and providing skilled care. You make a tremendous difference in the lives of the families you serve. Thanks for all you do.

And Mary, I'm sorry we failed you. I hope that next time around, you get the support and help you deserve.

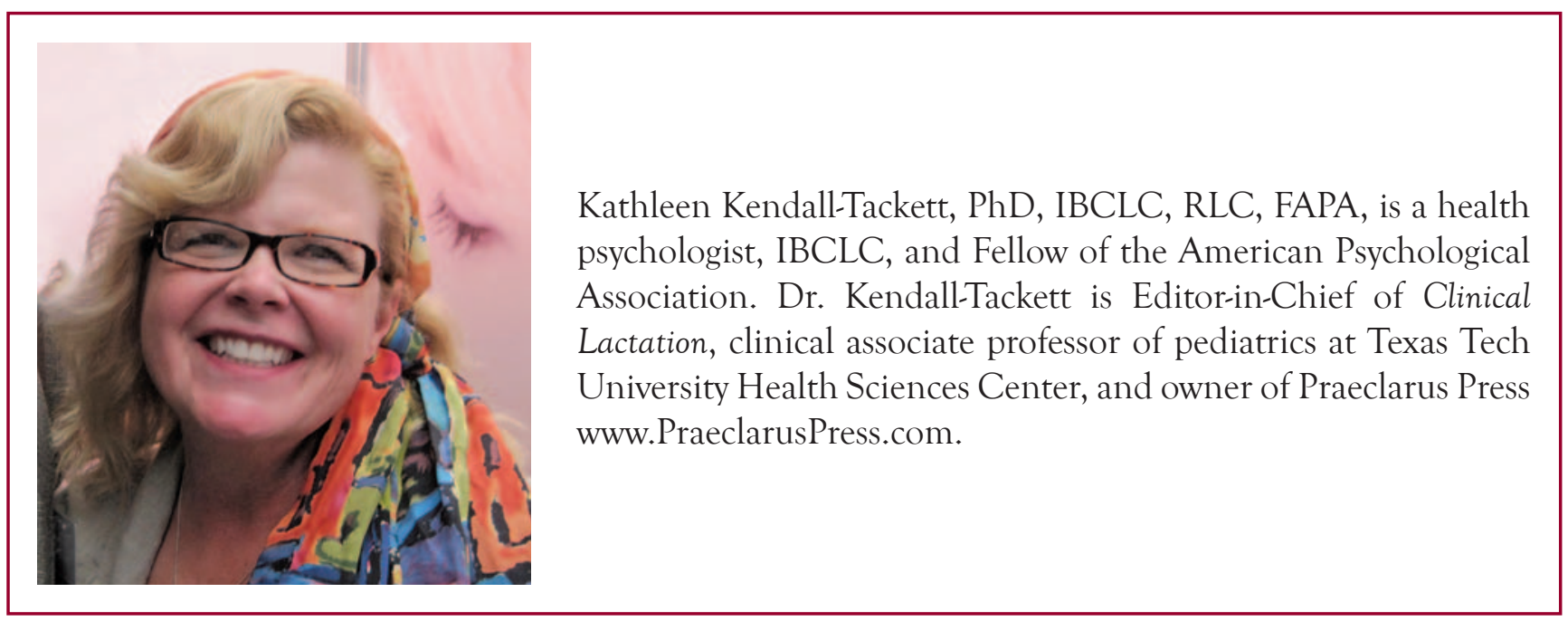

The Breastfeeding-Friendly Physician's Office: A New Clinical Protocol From the Academy of Breastfeeding Medicine

The Academy of Breastfeeding Medicine's new Clinical Protocol \#14: Breastfeeding-Friendly Physician's Office: Optimizing Care for Infants and Children, Revised 2013 presents 19 recommendations intended to improve physician support of breastfeeding initiation and duration. Among the recommendations are guidelines for establishing a written breastfeeding-friendly office policy, providing culturally and ethnically competent care related to lactation, introducing the subject of breastfeeding during the first trimester of pregnancy, allowing and encouraging breastfeeding in office waiting rooms, educating clinical physicians and staff on the benefits of breastfeeding, and encouraging enforcement and adoption of workplace laws that support breastfeeding.

Source: USBC 